OPEN ACCESS

Edited by:

Livio Casarini,

Università degli Studi di Modena e

Reggio Emilia, Italy

Reviewed by:

Erika Peverelli,

Università degli Studi di Milano, Italy

Francesco Poti,

Università degli Studi di Parma, Italy

*Correspondence:

Aylin C. Hanyaloglu a.hanyaloglu@imperial.ac.uk

Specialty section:

This article was submitted to

Reproduction,

a section of the journal

Frontiers in Endocrinology

Received: 24 September 2018

Accepted: 17 October 2018

Published: 02 November 2018

Citation:

Sayers N and Hanyaloglu AC (2018)

Intracellular Follicle-Stimulating

Hormone Receptor Trafficking and

Signaling. Front. Endocrinol. 9:653.

doi: 10.3389/fendo.2018.00653

\section{Intracellular Follicle-Stimulating Hormone Receptor Trafficking and Signaling}

\author{
Niamh Sayers and Aylin C. Hanyaloglu* \\ Department Surgery and Cancer, Institute of Reproductive and Developmental Biology, Imperial College London, London, \\ United Kingdom
}

Models of G protein-coupled receptor (GPCR) signaling have dramatically altered over the past two decades. Indeed, GPCRs such as the follicle-stimulating hormone receptor (FSHR) have contributed to these new emerging models. We now understand that receptor signaling is highly organized at a spatial level, whereby signaling not only occurs from the plasma membrane but distinct intracellular compartments. Recent studies in the role of membrane trafficking and spatial organization of GPCR signaling in regulating gonadotropin hormone receptor activity has identified novel intracellular compartments, which are tightly linked with receptor signaling and reciprocally regulated by the cellular trafficking machinery. Understanding the impact of these cell biological mechanisms to physiology and pathophysiology is emerging for certain GPCRs. However, for FSHR, the potential impact in both health and disease and the therapeutic possibilities of these newly identified systems is currently unknown, but offers the potential to reassess prior strategies, or unveil novel opportunities, in targeting this receptor.

Keywords: GPCR, FSH receptor, endocytosis, signaling, trafficking, cAMP, endosome

\section{INTRODUCTION}

The follicle-stimulating hormone receptor (FSHR) belongs to the superfamily of G proteincoupled receptors (GPCRs). With more than 800 members in humans they represent the largest family of signaling receptors and a major, successful, drug target (1). The canonical model of GPCR signaling is via plasma membrane localized receptors coupling to distinct heterotrimeric G proteins. However, we now understand the signaling pathways activated by GPCRs are much more complex to mediate the many distinct functions these receptors play in all physiological systems, but also equally important to decipher is how such signal pathways are regulated. These novel mechanisms are beginning to open up new avenues for therapeutic exploitation. One mechanism that not only contributes to the diversification of signaling but how cells decode or specify these signals is membrane trafficking. Classically, membrane trafficking was viewed as a mechanism to regulate sensitivity of a tissue to hormone, by altering the level of surface receptor either through ligand-mediated endocytosis in to the cell, and/or reduced biosynthetic trafficking of newly synthesized receptor. However, intracellular membrane compartments have been shown to represent additional signaling platforms for many kinds of receptors, including GPCRs such as FSHR. 
This review will discuss our current understanding of the molecular mechanisms and signaling roles of membrane trafficking of FSHR, and how gonadotropin hormone receptors have shed light on novel cell biological pathways potentially applicable to many GPCRs. We will primarily focus on postendocytic intracellular trafficking, and then discuss how this novel cell biology could shed light on specific facets of FSH/FSHR function and its implications to endocrine function.

\section{CLASSICAL REGULATION OF FSHR SIGNALING PATHWAYS}

\section{Pleiotropic G Protein Signal Profiles of FSHR}

FSHR is a member of the glycoprotein hormone receptor subfamily of the rhodopsin-like, or Class-A, family of GPCRs, which comprise a unique subgroup within the Class A family due to their leucine-rich repeat-containing extracellular ectodomain. Furthermore, the high glycosylation status of its ligand FSH, like other glycoprotein hormones, makes them the most complex of protein hormones. Glycoprotein hormones are heterodimers that consist of a common $\alpha$-subunit and a $\beta$-subunit that confers hormone specificity. These subunits are linked non-covalently and both subunits are subjected to $\mathrm{N}$-glycosylation that can alter their bioactivity (2). For FSH, two naturally occurring glycoforms have been identified, the hypo-glycosylated $\mathrm{FSH}^{21 / 18}$ and the fully glycosylated $\mathrm{FSH}^{24}$ (3), which have distinct activities [recently reviewed in (4)] and may be of significance to the trafficking pathways and intracellular signaling to be discussed below.

FSHR plays critical roles in reproduction, identified via numerous studies in both animal models and disease causing mutations in humans reviewed in Huhtaniemi and Themmen(5) and Jonas (6), but also extragonadal functions in uterus, adipose and bone, have been identified. In the gonads, FSH binds its receptor in testicular Sertoli cells and ovarian granulosa cells, where they regulate follicular development, steroidogenesis and spermatogenesis. Additional roles of FSHR in non-gonadal sites include myometrial contractility, regulation of lipid deposition, beiging and steroidogenesis in adipocytes and bone resorption functions in osteoclasts (7-10). The primary G protein pathway classically associated with the gonadally expressed receptor is the $\mathrm{G} \alpha_{\mathrm{s}} / \mathrm{cAMP} / \mathrm{PKA}$ (11). However, FSHR has also been shown to couple to additional $G$ protein pathways: $G \alpha_{\mathrm{q} / 11}$, which leads to activation of phospholipase $\mathrm{C}$, leading to production of diacylglycerol and inositol trisphosphate second messengers, the latter of which leads to increases in intracellular calcium levels. However, coupling of $\mathrm{G} \alpha_{\mathrm{q} / 11}$ to human FSHR is weaker than rodent FSHR and requires high receptor and hormone levels

\footnotetext{
Abbreviations: APPL1, adaptor protein containing PH domain; PTB domain, and Leucine zipper motif; $\beta 1 \mathrm{AR}, \beta 2 \mathrm{AR}$, beta adrenergic receptor 1 or 2 ; CCP, clathrin coated pit; EE, early endosome; EGF, epidermal growth factor; EGFR, EGF receptor; ESCRT, endosomal sorting complex required for transport; GIPC, Gai-interacting protein C terminus; GPCR, G protein-coupled receptor; LHR, luteinizing hormone receptor; PDZ, postsynaptic density 95/disc large/zonula occludens-1; PKA, protein kinase A; VEE, very early endosome.
}

(12-14). Recently it was reported that in pregnant myometrium FSHR levels are coupled to Gas to increase cAMP, a second messenger known to quiesce the myocytes, but during labor FSHR levels increase and the signaling switches to the procontractile calcium pathway, possibly by Gaq/11 coupling (7). Other $\mathrm{G}$ protein pathways can also be activated by FSHR. In Sertoli cells and osteoclasts FSHR has been reported to couple to $\mathrm{G} \alpha \mathrm{i} / \mathrm{o}$ family members, such as $\mathrm{G} \alpha_{\mathrm{i} 2}$, with subsequent MEK/Erk, NF- $\kappa$ B, Akt activation (9, 15, 16). Thus, like many GPCRs, FSHR exhibits the potential to activate $G$ protein signaling in a pleiotropic manner, and such diversity in $G$ protein signaling profiles may be tissue specific (Figure 1). The mechanisms underlying this tissue specificity in G protein coupling of FSHR may go beyond alterations in the levels of specific $\mathrm{G} \alpha$ subunits. Indeed, mechanisms such as GPCR homo and heteromerization and plasma membrane organization of receptors and signaling proteins such as in lipid rafts, are known to alter signal profiles of different GPCRs (17-19). FSHR is also subject to such mechanisms of signal diversity (20-22), although its role in directing tissue, or cell specific, responses is unknown.

\section{Arrestin-Dependent Desensitization, Internalization and Signaling}

The mechanisms mediating regulation of GPCR/G protein signaling are critical in shaping, or programming, the $G$ protein signal profile from the plasma membrane. Although models of this classic pathway of GPCR signal desensitization and internalization have rapidly evolved in the last 5 years, exhibiting increasing complexity, particularly as application of structural and super-resolution imaging are increasingly applied, the core features of this model (Figure 1) are still critical. Signal activation of GPCRs at the plasma membrane is regulated initially via a process of rapid desensitization, followed by internalization via clathrin-coated pits (CCPs). The initial step in this model involves receptor phosphorylation on serine/threonine residues by second messenger-activated kinases and/or GPCR kinases (GRKs). It is both the activated and phosphorylated receptor that enables recruitment of the adaptor protein arrestin from the cytoplasm. Arrestins are a family of adaptor proteins with an increasing array of functions, both for GPCRs and non-GPCR mediated signaling. The family contains four isoforms, where arrestin-1 and -4 are restricted to the visual system and are also termed "visual arrestins" $(23,24)$, whereas the other two isoforms, arrestin- 2 and -3 , also called $\beta$-arrestin- 1 and -2, are ubiquitously distributed and bind many GPCRs $(25,26)$. The arrestin-bound receptor desensitizes signaling via the uncoupling of receptor from its cognate $\mathrm{G}$ protein. Rapid arrestin-dependent internalization occurs by firstly inducing receptor clustering into CCPs, via its ability to bind receptor, clathrin heavy chain and core clathrin adaptor proteins (namely the $\beta 2$ subunit of AP2) (27). This model, comprehensively described across many reviews, have discussed ways in which GPCRs may employ this system in distinct manners, such as differential phosphorylation of intracellular GPCR domains, primarily at the carboxy terminal tail (C-tail), alters association kinetics of GPCR to arrestin and its subsequent impact on receptor activity $(28,29)$. However, 


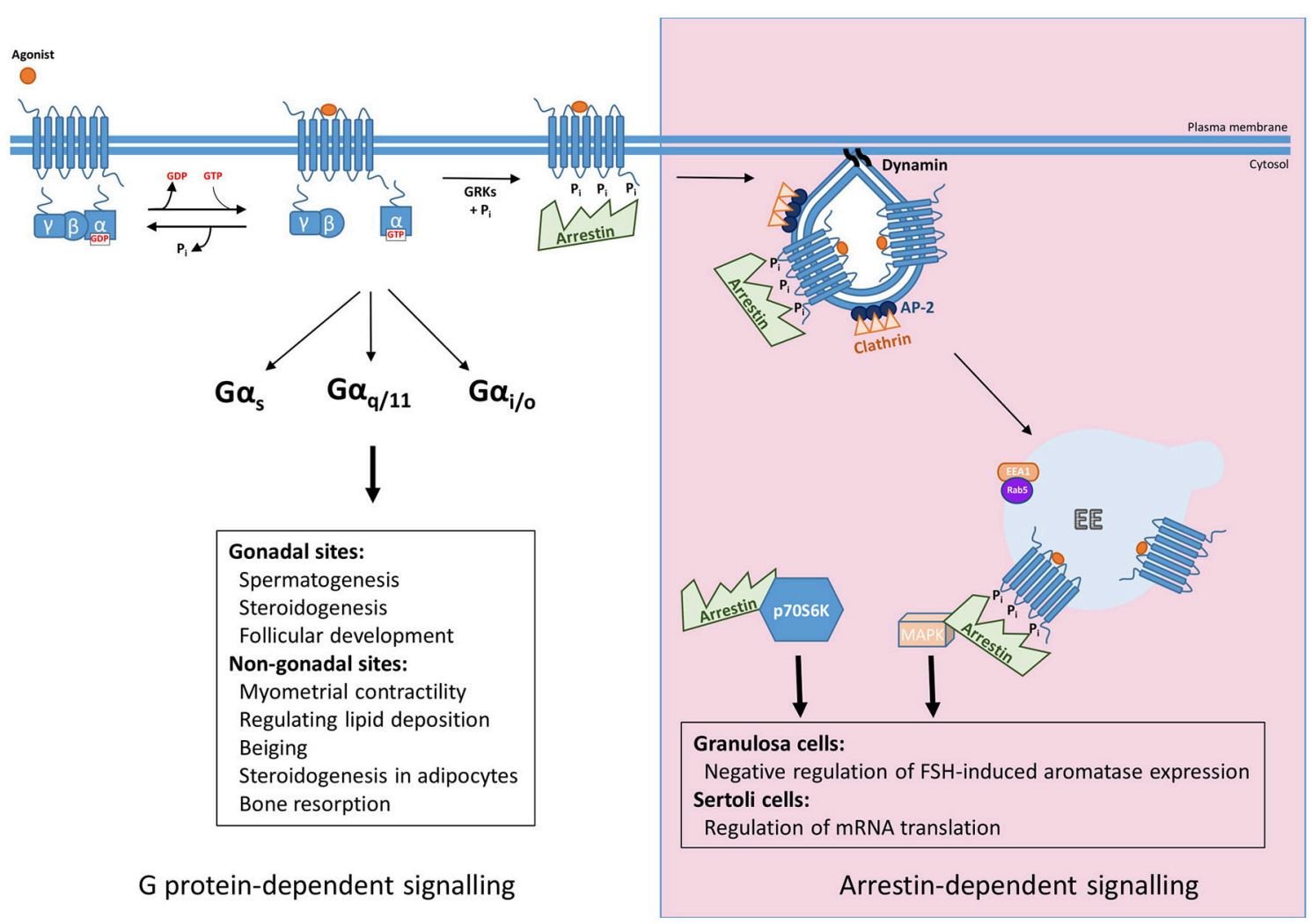

FIGURE 1 | Summary of G protein and arrestin-mediated signal pathways activated by FSHR. Upon ligand binding FSHR has been reported to couple to GaS, $\mathrm{G} \alpha \mathrm{q} / 11$, and Gai/o heterotrimeric G proteins to mediate its downstream effects, both those in the gonads such as spermatogenesis, steroidogenesis, and follicular development, and more recently at non-gonadal sites, see text for further details. The archetypal view of GPCR signaling occurs in a G protein-dependent manner, however, agonist-activated, phosphorylated receptor recruits arrestin, for $\mathrm{G}$ protein signal desensitization and internalization via clathrin-coated pits. Furthermore, arrestin mediates signaling pathways independent of $G$ proteins. This can occur through the scaffolding protein binding signaling proteins such as components of MAPK pathways after G protein activation, or independent of FSHR by complexing and activating ribosomal protein, p70S6K.

more recent structural and functional studies demonstrating the distinct modalities that arrestin can complex with GPCR and the G protein bound GPCR (30-34) have unveiled novel features of regulation that could be highly pertinent for FSHR, and will be discussed below.

FSHR signaling is regulated by this canonical GRK/arrestin model. As discussed above, internalization and desensitization are mediated by GRK phosphorylation, followed by arrestin binding. This is true for FSHR, where a cluster of five serine/threonine residues in its C-tail were identified as the key sites for GRK 2, 5, and 6 phosphorylation (35). Interestingly, while GRK 2 is predominantly involved in FSHR arrestinmediated desensitization, GRKs 5 and 6 also promote arrestin binding but for scaffolding signaling proteins and signal activation (36). For FSHR, specific threonine residues in the third intracellular loop have been identified to dictate their rate of internalization and arrestin sensitivity, or binding (37). Further, there are differences in how FSHR engages with the GRK/arrestin mechanism between rodent and human receptors and also when compared to its "sister receptor" the $\mathrm{LH}$ receptor (LHR) that has unveiled specific structural motifs in FSHR involved in its internalization rate. The rodent
FSHR internalizes faster than the rodent LHR and the human FSHR. Creating chimeras between receptors revealed that six amino-acids in transmembrane 4, intracellular loop 3 and transmembrane 7 determine internalization of rodent FSHR to be 3 times faster than human FSHR, and its sensitivity to arrestins in enhancing receptor internalization (38). In addition, a serine/threonine phosphorylation cluster in the rodent FSHR $\mathrm{C}$-tail is also involved in arrestin binding and arrestin-dependent internalization and desensitization, but not MAPK signaling (36). The importance of the third intracellular loop and Ctail residues in arrestin binding is interesting especially given recent key developments in our molecular understanding of how arrestin engages with GPCRs to mediate its distinct functions. Structural studies have now shown that arrestin engages active receptor in at least three forms, via the phosphorylated C-tail, the receptor core, or both. These forms have helped to explain how arrestin can mediate $G$ protein uncoupling, internalization and yet also facilitate signaling when either as a stable or transient GPCR/arrestin complex $(30,31,33,39)$. These differences in arrestin binding to receptor could suggest that for FSHR core and C-tail interactions with arrestin may mediate its functions in an opposing manner than described for other GPCRs. However, as 
will be covered in the next section, arrestins also have roles in driving gonadotropin hormone receptor signaling and there may be alternate FSHR/arrestin conformations to mediate its various functions.

The role of arrestins as scaffolding proteins for different signaling proteins is now well recognized. The primary signaling pathway studied that is activated by such arrestin scaffolds is the MAPK pathway (40-42). For FSHR, ligand-dependent ERK signaling exhibits a sustained profile whereby the early activation is dependent on Gas/PKA activation while the sustained response requires arrestins [(36) and Figure 1]. Likewise, the ligand-dependent interactions between FSHR and arrestin are also sustained as measured by BRET (43), although there have been no image-based data confirming sustained, or internalized FSHR/arrestin complexes in cells. Given arrestins are involved in FSHR internalization, its role in signaling strongly suggests additional scaffolding roles of this adaptor protein, and/or a requirement of receptor internalization of ERK signaling as has been demonstrated for LHR (44). However, a partial inhibition of FSHR internalization via arrestin and dynamin dominant negative mutants did not impact ERK signaling (45). Although more work is required to clarify how arrestins mediate G protein-independent FSH signaling, perhaps recent studies could provide clues to a mechanistic understanding of how FSHR mediates sustained arrestin-dependent MAPK responses. The $\beta 1$-adrenergic receptor ( $\beta 1 \mathrm{AR})$ is a GPCR that induces a sustained ERK profile mediated by arrestins, but only transiently associates with arrestin at the plasma membrane, while arrestin remains associated within CCPs in order to activate signaling in a sustained manner (46). The authors demonstrated that arrestin transiently binds to the core of $\beta 1 \mathrm{AR}$ only, which induces a conformational change in arrestin resulting in its capture by binding phosphoinositides in the plasma membrane and clathrin associated adaptor proteins (33). Given the sustained ERK signaling profile of FSHR, that the third intracellular loop residues are involved in arrestin binding, while C-tail sites do not regulate ERK signaling $(37,47,48)$ and further similarities in these receptors with the $\beta 1 \mathrm{AR}$ in their post-endocytic pathways [see section Postendocytic sorting and endosomal signaling of FSHR from a novel compartment; the very early endosome (VEE)], it is possible that arrestin engages with FSHR in a similar manner. Alternatively, or in addition to this receptor-independent arrestin signaling complex, is the recent elegant report demonstrating that FSHR activates p70S6K within an arrestin complex constitutively assembled with a p70S6K/ribosomal protein S6 (rpS6) to regulate mRNA translation (49). This study supports recent structural findings that arrestin, after its dissociation from receptor, can maintain various active conformations (50), but also suggests that receptor-independent "active" arrestin complexes may not only be a feature of CCPs but also other subcellular locations.

The ability of GPCRs to activate more than one pathway of signaling, such as $\mathrm{G}$ protein and arrestin-mediated signaling via the stabilization of a certain active conformation of the receptor, is termed signaling bias (28) and is of high pharmacological interest. Bias can occur through different ligands (ligand bias) or even receptor mutations (receptor bias) (51). From a therapeutic perspective, the ability to specifically target the desired cellular effects, through one pathway, without activating unwanted side effects has been shown to be a feasible strategy for certain GPCRs. In terms of G protein vs. arrestin-mediated signaling, recent studies have challenged this model for certain GPCRs, suggesting that $G$ protein activation is still an essential upstream event of arrestin-dependent ERK signaling $(52,53)$. Perhaps an argument against a requirement for $G$ protein activation in arrestin-mediated signaling for FSHR is via the observation that lowering expression levels of the receptor to a level where there is no detectable cAMP signaling can induce bias to arrestindependent signaling (54). This was first revealed by studies on the A189V FSHR mutant, which is expressed at very low levels on the cell surface and is non-functional with respects to Gas/cAMP signaling (55). Yet, when both A189V mutant and wild-type FSHR are expressed at equivalent low levels they are only able to trigger G-protein independent MAPK activation (54).

For biased signaling to be therapeutically explored for FSHR, there must be a well characterized understanding of the in vivo role of arrestin in FSHR signaling. So far, it has been demonstrated that in Sertoli cells arrestin may regulate mRNA translation and a possible negative regulation of FSH-induced aromatase expression in rodent granulosa cells (via manipulation of GRK6 levels as an upstream step in arrestin binding) (56) (Figure 1). This latter study is perhaps corroborated by findings in an immortalized human granulosa tumor cell line, whereby arrestins negatively regulate Gas/cAMP/PKA pathway, not in terms of classical desensitization, as in these cells gonadotropinmediated ERK signaling via arrestins was evident in the absence of cAMP signaling. Specifically, FSH, but not LH, dependent apoptosis occurred by cellular depletion of arrestins, due to increases in cAMP/PKA signaling, thus suggesting a role for arrestins in regulating balance between cell proliferation and apoptosis (57). While promising, further work needs to be conducted to evaluate the potential benefit of arrestin-based biased agonism.

\section{Post-endocytic Intracellular Trafficking Pathways of FSHR}

Following internalization, GPCRs are trafficked to endosomes where they are sorted to either a plasma membrane recycling pathway, or to the lysosomal pathway for degradation. Such pathways program the temporal profile of $\mathrm{G}$ protein signaling, by regulating resensitization/hormone recovery (recycling) or permanent signal termination (degradation), Additionally, the sorting fate of a GPCR pharmacologically manipulated and altered in disease (58). However, we now know that the endocytic system does not only regulate the surface density of receptors but that these divergent, and complex, sorting pathways have direct roles as platforms for signaling, including $G$ protein signaling (59-61). This will be further discussed in section Postendocytic sorting and endosomal signaling of FSHR from a novel compartment; the very early endosome (VEE). The mechanisms that underlie these divergent post-endocytic fates are tightly regulated at multiple levels and are interlinked with the receptors own signaling. These complex mechanisms have 
recently been reviewed by us and others (61-64) and will not be described in detail here except to illustrate core features that enable discussion of current understanding of FSHR sorting and intracellular signaling.

The textbook model of cargo sorting depicts the Rab5 early endosome (EE) as the common post-endocytic compartment from which receptors are first sorted to opposing fates. GPCRs sorted to a degradative pathway following internalization are trafficked from EEs to Rab7 positive late endosomes. Receptors are involuted into vesicles within the lumen of these endosomes, to form multivesicular bodies (MVBs). MVBs will then fuse with lysosomes resulting in protein degradation. GPCRs will engage with this pathway with distinct kinetics and for those receptors targeted to the recycling pathway, chronic ligand stimulation will reroute receptors to this degradative pathway as part of the mechanism of downregulation. Classically, lysosomal targeting of different receptors is via ubiquitination at lysine residues and engagement with endosomal sorting complex required for transport (ESCRT)-dependent degradation, however, GPCRs exhibit ubiquitin-independent and ESCRT-independence in their mechanisms of degradation [reviewed in $(65,66)]$. GPCRs targeted to a rapid recycling pathway are sorted from EEs to Rab4 positive recycling endosomes. An important feature of GPCRs targeted to recycling pathways, is that this is regulated by interactions with specific sequences in the GPCR C-tails, also termed sequence-directed, or regulated, recycling. This mode of recycling is distinct from recycling of other kinds of membrane cargo, e.g., transferrin receptor that does not require its C-tail for recycling and occurs with the bulk membrane flow (default recycling). The distal C-tail receptor sequences are not only essential for recycling, but if fused to the carboxy-terminus of a GPCR sorted to a degradative pathway, it will reroute that GPCR to the recycling pathway. There are no common sequences that determine whether any GPCR undergoes regulated recycling, as they are highly divergent. However, several recycling sequences identified, such as first identified with the $\beta 2$-adrenergic receptor $(\beta 2 \mathrm{AR})$, correspond to a type 1 (PSD95)/discs large (Dlg)/zonula occludens-1 (Zo-1) (PDZ) binding sequence or "PDZ ligand," specifically $\mathrm{S} / \mathrm{T}-\mathrm{X}-\Phi$, (where $\Phi$ is any hydrophobic residue) $(67,68)$. PDZ proteins are scaffold proteins and for GPCRs that bind PDZ proteins, they are often able to bind more than $1 \mathrm{PDZ}$ protein (69), suggesting these sequences and interactions may have additional functions to directing receptors to the recycling pathway. For the $\beta 2 \mathrm{AR}$ the interacting PDZ-domain containing protein partner responsible for recycling is the endosomally localized PDZ protein, sorting nexin-27 (SNX27) (70). As mentioned above, these recycling sequences are very distinct amongst receptors, so there are several examples of GPCRs targeted to a recycling pathway that do not contain PDZ type 1 ligands or any other recognizable motif, and hence for many their corresponding interacting protein partners are unknown $(58,71)$. This is also the case for the FSHR whereby both rodent and human FSHR are recycled back to the plasma membrane via specific $\mathrm{C}$-tail sequences that does not indicate any potential binding partners such as a PDZ protein (72). More recently a role for palmitoylation in FSHR sorting has been proposed (73). In FSHR it is known that there are 2 conserved and 1 non-conserved cysteine in the FSHR C-tail that were all palmitoylated, but only 1 of the conserved cysteines (cysteine 629) affected receptor function by impairing cell surface expression (74). A follow up study demonstrated that mutation of all three cysteines to glycine significantly impaired biosynthetic trafficking of the receptor to the plasma membrane and thus exhibited reduced signaling. Interestingly, the receptor that was transported to the plasma membrane exhibited similar internalization kinetics but impaired recycling, the receptor thus being routed to the degradative pathway (73). This suggests that altering palmitoylation of the receptor changes the ability of the $\mathrm{C}$-tail, and presumably the distal recycling sequence, to interact with key machinery that mediates its sorting, but also indicates that a cell could alter FSHR trafficking, and subsequently signaling responsiveness, through alterations in these post-translation modifications.

While the above describes that the recycling pathway of FSHR, and other GPCRs, involves a one-step mechanism with its recycling sequence and interacting partner, which may be unique to a given GPCR, we now know that both common and receptor-specific post-endocytic mechanisms exist. Furthermore, sequence-directed recycling (and lysosomal sorting) occur via a complex, multi-step system, with the GPCR's own signaling playing a key role in driving receptors to these distinct cellular fates, in addition to trafficking regulating the signal from that receptor. This interconnected property of post-endocytic trafficking and GPCR signaling has been highlighted recently through studies of the gonadotropin hormone receptors and will be discussed next.

\section{POST-ENDOCYTIC SORTING AND ENDOSOMAL SIGNALING OF FSHR FROM A NOVEL COMPARTMENT; THE VERY EARLY ENDOSOME (VEE)}

The internalization of GPCRs into the endocytic network is no longer viewed as a mechanism to only control plasma membrane signaling but to also provide additional platforms to continue or reactivate signaling from intracellular compartments, including $G$ protein signaling. Furthermore, this spatial control of signaling has been shown to be important for cells to decode common second messenger signaling molecules, such as cAMP signaling, activated by many GPCRs, into specific downstream cellular functions (59-61). Intracellular signaling is also important for the FSHR, however, it is through studies on human LHR has unveiled how important, and tightly regulated, the compartmentalization of receptors within the complex endomembrane network is to receptor endosomal signaling.

\section{Discovery of the VEE; A Tale of Serendipity}

Many GPCRs, including the human FSHR and LHR undergo the regulated recycling pathway described in Section Postendocytic intracellular trafficking pathways of FSHR. Our studies first identifying the VEE with the gonadotropin hormone receptors were initially driven as way to understand why GPCRs have distinct recycling sequences if there is a common 
primary function, i.e., sorting to the recycling pathway. Thus, comparisons were first made between the $\beta 2 \mathrm{AR}$ and LHR that initially seem quite similar in their signaling and trafficking profiles (44). Both are Gas-coupled receptors that internalize via arrestin/clathrin pathways and undergo sequence-directed recycling. However, they have distinct $\mathrm{C}$-tail recycling sequences and bind different PDZ proteins for their sorting, SNX27 for $\beta 2 \mathrm{AR}$ and GIPC (Gai-interacting protein, C-terminus) for LHR $(68,70)$. Unexpectedly, when agonist-induced LHR endocytosis was monitored by live confocal microscopy imaging it was evident this receptor trafficked to endosomes closer to the plasma membrane that were approximately a third of the diameter of endosomes containing $\beta 2 \mathrm{AR}$. FSHR and the $\beta 1 \mathrm{AR}$ also internalized to these small endosomes. The identity of this compartment, in terms of the proteins or adaptors that traffic there are poorly understood, except they are distinct from those classically found in the EE and EE intermediates such as EE antigen 1, phosphatidylinositol-3 phosphate (a lipid enriched in the EE membrane) and Rab5 (44). As it was previously established that LHR sorting to the recycling pathway required its C-tail interaction with the PDZ protein GIPC, the hypothesis was that this protein interaction also directed LHR to these small endosomes. Indeed, truncation of the LHR recycling sequence or knockdown of GIPC levels inhibited recycling and also rerouted receptor to the larger EEs (Figures 2A,B). As interactions with GIPC occurred only early on during receptor clustering in to CCPs, further supported a model whereby LHR recycling must occur from these small endosomes, we then termed very early endosomes (VEEs) (44). While the discovery of a new cellular compartment was very unexpected, our original aim of understanding the role of these different GPCR recycling sequences was also in part addressed, as it highlights these sorting sequences may encode functions at distinct steps in the endocytic trafficking of a GPCR, and not only sorting from endosomes. In this case, it was sorting to distinct populations of endosomes. It is well known that trafficking regulates signaling, thus what were the signaling functions of the VEE? Surprisingly, when LHR trafficking was rerouted away from VEEs to EEs, via inhibiting interaction with GIPC, the ligand-induced CAMP signaling was not affected but ERK signaling profile was altered from a sustained to a transient one (Figure 2B) (44). Given that both acute and sustained ERK signaling was dependent on internalization, suggests that under conditions when GIPC is depleted, the receptor is rapidly routed through the VEE, but not maintained in this compartment due to its trafficking to EEs, hence the altered temporal ERK signaling profile (see Figure 2B). It also highlights the interconnectivity of endomembrane systems; indeed, we also demonstrated in this study that the transferrin receptor can internalize through the VEE on its way to the EE. Intriguingly, the ligand-induced ERK signaling of $\beta 1 A R$ and FSHR was also affected by GIPC knockdown. While $\beta 1 \mathrm{AR}$ is known to interact with GIPC via its C-tail (75), this result was unexpected for FSHR since its recycling sequence contains no PDZ ligand and there are no prior reports of its interaction with GIPC. However, FSHR is known to directly interact with the adaptor protein containing $\mathrm{PH}$ domain, PTB domain, and leucine zipper motif (APPL1) $(12,76,77)$, and so far, APPL1 is the only protein identified that localizes to a subpopulation of VEEs. Given APPL1 can directly bind GIPC (78) this may underlie the GIPC-dependent nature of ERK signaling by FSHR.

\section{The Role of APPL1 in FSHR and VEE Function}

To date, APPL1 is the only known protein present on the VEEs, as the PDZ protein GIPC only associates during the very early steps of endocytosis at the CCP (44). APPL1 is a well-studied adaptor protein comprised by multiple protein and membrane interacting domains (79). Prior studies have shown it localize to EE Rab5 compartment but at an intermediate step prior to conversion of endosomes to EEA1 positive endosomes (80). APPL1 is reported to localize to other compartments including vesicles that do not have Rab5 (81), akin to what we have observed with the gonadotropin hormone receptors and the VEE (44). APPL1 displays multiple, and integrative functions in cargo trafficking and receptor signaling, as evident from the numerous reported interactions including Rabs, receptors such as FSHR, EGF receptor, insulin receptor, adiponectin receptor, androgen receptor, kinases and phosphatases, like protein kinase $\mathrm{B}$ and PtdIns(3)P kinase, and PDZ proteins like GIPC (82). Prior to our reports on the VEE, APPL1 was shown to form a complex with FSHR, not via the C-tail but via three specific residues in its first intracellular loop (76). In addition, this complex contained additional adaptor and signaling proteins, including APPL2, Akt, and FOXO1. Such a complex was shown to propagate FSH-induced $\mathrm{PI} 3 \mathrm{~K} /$ Akt signaling, $\mathrm{IP}_{3}$ production and calcium release $(12,76,77,83)$. This is consistent with APPL1's roles in positive regulation of signaling (78). These signal pathways in FSHR/APPL1 complexes remain to be studied in the context of the VEE where focus so far has been primarily on cAMP and MAPK.

APPL1 plays critical roles in gonadotropin receptor trafficking and endosomal cAMP signaling from the VEE (84). As described above, interactions with GIPC are essential for directing receptors to the VEE (Figure 2B). In contrast, APPL1 is not required for GPCR localization to the VEE but is essential for LHR and FSHR recycling (Figure 2C). Interestingly, it is the receptor's own cAMP/PKA signaling that drives this APPL1-dependent recycling (60). The mechanism underlying this requirement is that $\mathrm{LH}$-mediated PKA activation must phosphorylate APPL1 at serine 410 for the receptor to recycle back to the plasma membrane. This raised the possibility that LHR activates signaling from the VEE as it would provide a means for high localized regulation of APPL1 (phosphorylation) at the endomembrane, and perhaps specific populations of APPL1. Inhibiting internalization of LHR almost completely abolished ligand induced increases in cAMP, a finding corroborated using a nanobody biosensor that recognizes active Gas (85), which localized to a subpopulation of LHR endosomes (84). If the primary location of cAMP signaling from these receptors was the VEE, then inhibition of recycling should enhance cAMP signals. Indeed, this is the case when cellular levels of APPL1 are depleted for all known VEE-targeted receptors (LHR, FSHR, and $\beta 1 \mathrm{AR}$, see Figure 2C). However, 


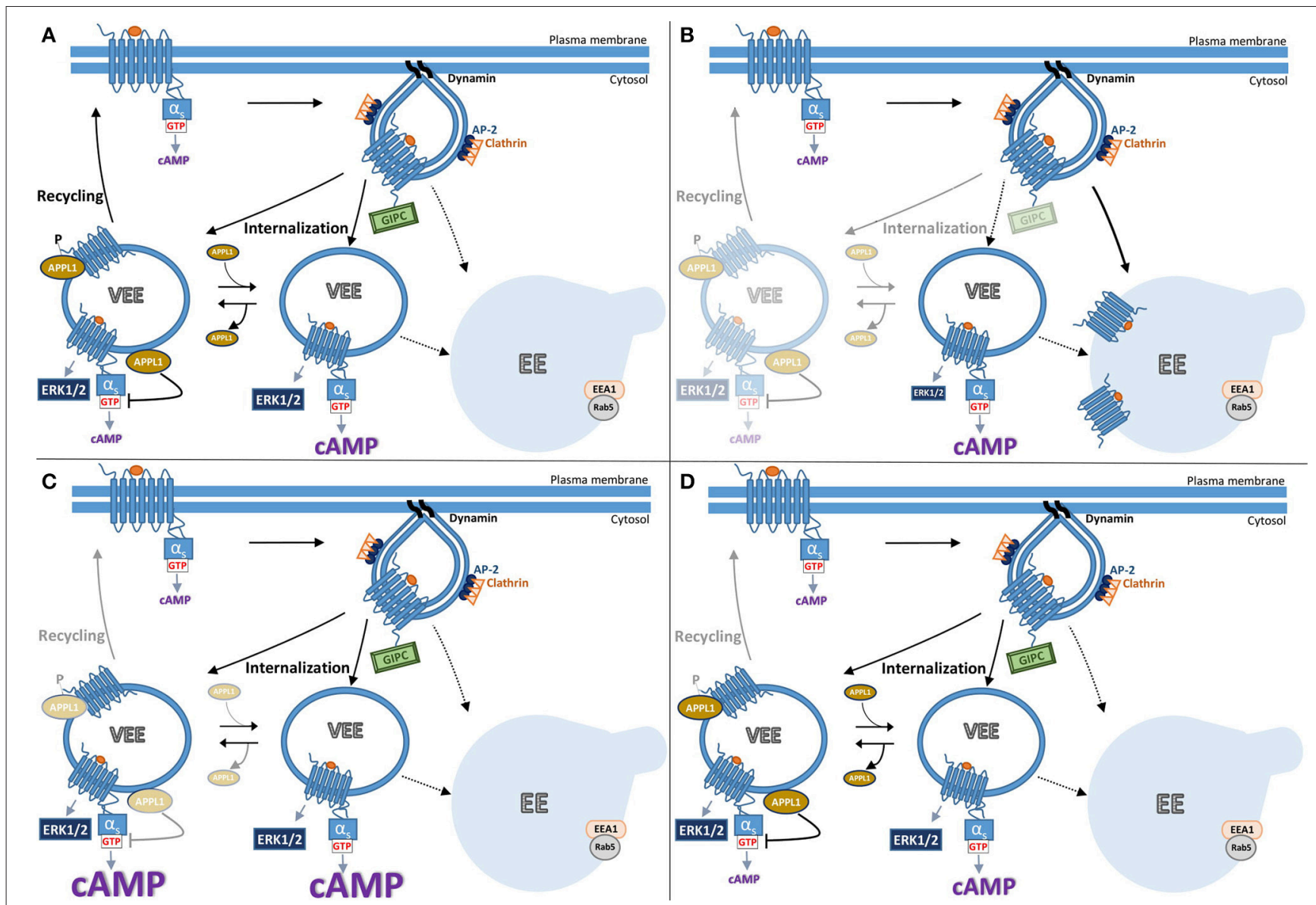

FIGURE 2 | Model summarizing current understanding of gonadotropin hormone receptor post-endocytic pathways and receptor signaling from VEEs. The trafficking of FSHR and LHR to the VEE is inextricably linked to the receptor's signal output, whereby the manipulation of which at distinct steps results in different trafficking and signal profiles. (A) Following ligand-activation FSHR internalizes to the very early endosome (VEE). The VEE differs from the early endosome (EE) in its smaller size and neither contain EEA1 nor Rab5, classical markers for the EE. During receptor-mediated endocytosis of FSHR into a clathrin-coated pit, the PDZ domain protein, GIPC, is recruited at the cytosolic interface of the GPCR. Receptor then enters the complex endosomal network where it is primarily localized to the VEE. There are two types of VEE depicted, one contains the adaptor protein, APPL1 (see text), and one is without. GIPC dissociates before FSHR enters the VEE. From the VEE receptor is able to elicit downstream signaling cascades, including CAMP generation and ERK1/2 activation. This CAMP/PKA signal phosphorylates APPL1 on Serine 410. Receptor is trafficked to APPL1 positive VEEs, where the unphosphorylated APPL1 negatively regulates endosomal CAMP signaling. The phosphorylated APPL1 is required for receptor recycling back to the plasma membrane. (B) The receptor can be rerouted from the VEE to the EE by the loss of GIPC or disruptions in the receptors ability to interact with this PDZ protein. Loss of GIPC results in the trafficking of the receptor from the VEE pathway to the EE and loss of plasma membrane recycling. While endosomal cAMP signaling is not affected, ERK signaling profile is more transient as the receptor only rapidly passes through the VEE to the EE. (C) Loss of APPL1 does not alter the endosomal organization of the receptor but inhibits recycling. The "trapped" receptor in the VEE compartment results in increases in endosomal CAMP signaling due to APPL1's role in negative regulation of G protein signaling, but without impacting ERK signal profile. (D) Manipulating the ability of APPL1 to be phosphorylated on Serine 410, either by inhibition of PKA activity or mutation of serine 410 to alanine, specifically inhibits recycling but not cAMP endosomal signaling.

ERK signaling (strength and kinetics) is not affected under these conditions and inhibition of LHR recycling via a PKA inhibitor (as APPL1 phosphorylation is needed for recycling) has no effect on LHR-cAMP signaling. Thus, APPL1 has an additional role in negatively regulating cAMP from VEE targeted GPCRs (84). How APPL1 controls GPCR/Gas coupling is still unknown, yet intriguingly it is the unphosphorylated form of APPL1 that mediates this, indicating that distinct populations of APPL1, phosphorylated and unphosphorylated forms, have opposing functions on VEE-targeted receptors (Figure 2). Furthermore, it highlights that endosomal signaling must be "switched off" prior to receptor recycling, as has been shown in GPCR endosomal signaling from the $\mathrm{EE}$ and the role of the retromer complex $(86,87)$. Overall, the VEE-network displays exquisite control of signaling and trafficking via the actions of APPL1. There are many outstanding molecular questions for this system, including if there are any roles for the related protein APPL2, which has both common and distinct functions to APPL1, especially as FSHR forms a complex with both adaptor proteins (77). Are other signal pathways that FSHR is known to activate, such as Gai signaling (see section Classical regulation of FSHR signaling pathways), also regulated at the level of the VEE and by APPL1? Another question these studies have raised is why is such complexity within the endosomal system required? One 
possibility is that it enables a cell to alter receptor activity at many levels and potentially in a pathway specific manner (location bias in signaling), perhaps in response to physiologically relevant changes in its extracellular environment (e.g., dynamic hormonal environment during menstrual cycle) to pathological changes in disease. This is illustrated in Figure 2, whereby altering levels of key adaptor proteins GIPC and APPL1, alters receptor signaling to ERK or cAMP respectively (Figures 2B,C), or at the level of APPL1 regulation of PKA mediated phosphorylation, would result in a distinct trafficking and signal phenotype (Figure 2D).

Overall, compartmentalization of internalized gonadotropin hormone receptors, and indeed many other GPCRs, mediates their signal activity at specific intracellular sites, and represents a mechanism for cells to diversify signaling even from the same pathway (e.g., cAMP/PKA) to possibly distinct functional consequences. It also raises the intriguing possibility that GPCR activity, can be reprogrammed by the cell to alter the compartment the receptor is targeted to via altering expression of key proteins in this pathway, e.g., GIPC, APPL1 (Figure 2). Such a model may explain how FSHR activity in the gonads, commonly mediated by cAMP/PKA, underlies distinct functions but also specificity of this pleiotropically coupled GPCR.

\section{OUTSTANDING QUESTIONS AND FUTURE PERSPECTIVES}

These recent advances in FSHR function from its distinct roles in extragonadal tissues and novel cell biological functions demonstrates that FSHR is a good example of a GPCR that achieves signal diversification via multiple strategies and a prototype receptor for understanding novel facets of GPCR function. Whether such pathways are then perturbed in disease and if we can harness these properties of GPCRs therapeutically needs to be addressed.

While much has been uncovered about novel intracellular trafficking systems, there are numerous outstanding questions at the molecular level. A major one to be tackled in the future is the identifying the molecular composition (both protein and lipid content of the membrane) of the VEEs, as so far APPL1 is the only known protein to reside there and is only present in $\sim 50 \%$ of the VEE population (84). While not a trivial task to unpick, recent developments in proteomics such as the application of engineered ascorbic acid peroxidase (APEX)mediated approaches that can capture the local protein network of a receptor with high spatial-temporal resolution as it traffics through the endocytic system (88), may be able to uncover specific VEE proteins and whether such proteins are core to these endosomes, or receptor-specific. A critical outstanding question is understanding the downstream role of VEE targeting, and could there be any clues from disease-causing mutations in the gonadotropin hormone receptors? It is likely that the VEE and APPL1-dependent regulation is a conserved mechanism across cells, as for many membrane trafficking pathways. In primary human endometrial stromal cells, LHR could traffic to VEEs and recycle in an APPL1-dependent manner (84), although the downstream functional role/s in the endometrium, a tissue reported to express both functional LHR and FSHR are unknown. A role for APPL1 in regulating gonadotropin action has been reported in the ovary whereby knockdown of APPL1 in bovine theca cells enhances LH-mediated androgen production (89). Given loss of APPL1 increases ligand-dependent cAMP signaling in HEK 293 cells (84) (Figure 2C), is consistent with this finding and could suggest that deregulated signaling by VEE/APPL1 has key physiological/pathophysiological roles in the ovary such as steroidogenesis and conditions where there is enhanced LH activity e.g., PCOS. Indeed, a direct role for gonadotropin hormone receptor endocytosis in LH-mediated cAMP signaling in the mouse ovarian follicle and the resumption of meiosis in the oocyte has been demonstrated (90). Specific roles for FSH/FSHR function will need to be investigated, given there are known disease causing mutations that alter FSHR signal desensitization and inhibit internalization (91). The reassessment of known mutations in the context of the VEE would be highly informative as tools for further understanding how FSHR engages with these intracellular trafficking and signaling mechanisms. However, such disease-causing mutations are rare, and from a translational perspective there would be value in assessing known single nucleotide polymorphisms that impact FSHR activity for potential alterations in VEE function. For example, the FSHR asparagine/serine polymorphism at position 680 (N680S), whereby in the general population approximately $60 \%$ are $\mathrm{N} 680$ and $40 \%$ S680, while in infertile individuals this is more 50/50. This SNP determines poorer responsiveness to FSH in women bearing the $\mathrm{N}$ variant compared to the $\mathrm{S}$ carriers that has been linked to temporal alterations in cAMP, ERK1/2 and CREB responses in human granulosa cells, whereby interestingly it is the $\mathrm{N}$ variant that exhibits faster signal properties $(92,93)$. These altered signal properties could result in altered arrestinmediated signaling and/or altered sorting of FSHR to endosomal compartments. Although any potential alterations in intracellular trafficking at the level of the VEE are unknown, given the Ctail location of the SNP, located upstream of the distal recycling sequence of FSHR (72), one could predict that this variant may modulate interactions with protein partners that bind this distal recycling sequence, resulting in distinct post-endocytic fates and altered endosomal compartmentalization. The faster kinetics in signaling exhibited by the $\mathrm{N}$ variant may indicate for example, a lack of APPL1-dependent regulation in signaling and/or inability of GIPC to direct the receptors to the VEE. Given the more widespread functions of FSH that have been reported, this SNP may be predictive of other conditions, such as pre-term birth whereby carriers homozygous for the $\mathrm{N}$ variant, as evaluated from placental samples, had a significantly higher risk of preterm birth than the $S$ variant (94). This variant is not only of significance to female health but also to males. Infertile men carrying the $680 \mathrm{~N}$ variant compared to the $680 \mathrm{~S}$ respond better to FSH treatment as assessed by improved DNA fragmentation index of their spermatozoa (95). Furthermore, the increasing reports of FSHR in endothelial cells, bone and adipose $(8,9)$ may unveil future roles for altered spatial control of FSHR signaling in cancer, obesity, type 2 diabetes and osteoporosis.

The ability to pharmacologically exploit these new GPCR signaling models and target the intracellular signaling receptor 
specifically has been recently demonstrated for three distinct GPCRs. Employing cholestenol-conjugated antagonists, which accumulate in the lumen of endosomal compartments, can block endosomal signaling from the neurokinin 1 receptor and calcitonin-gene related peptide receptor and has been shown to be an effective nociceptive target in animal models $(96,97)$. Whilst a similar strategy to inhibit endosomal signaling of the protease-activated receptor 2 prevented hyperexcitability of pain receptors in the colon and thus has been proposed to be of therapeutic value in pain management of irritable bowel syndrome (98). These studies set an important precedent for the likely success of targeting intracellular signaling of GPCRs, however, we know GPCRs including FSHR exhibit pleiotropic signaling and the ability to also target intracellular signaling more specifically, either at a pathway level or endosomal compartment level (e.g., VEE vs. EE) could be valuable. In other words, to create biased intracellularly targeted compounds may offer avenues for more efficacious compounds with less side effects. For FSHR there may already exist avenues to develop such ligands, as a number of small molecule, orally available, and cell permeable, compounds have been produced (99), although their role in altering intracellular signaling of FSHR remain to be determined. It may not always be necessary to target intracellular receptors, and perhaps altering the endosomal fate of a receptor by targeting the plasma membrane receptor prior to its internalization may be advantageous, to also induce location bias. This could even be achieved through native ligands; for FSHR there are known glycovariants of FSH that exhibit distinct activities (4).

\section{REFERENCES}

1. Hauser AS, Attwood MM, Rask-Andersen M, Schioth HB, Gloriam DE. Trends in GPCR drug discovery: new agents, targets and indications. Nat Rev Drug Discov. (2017) 16:829-42. doi: 10.1038/nrd.2017.178

2. Green ED, Baenziger JU. Asparagine-linked oligosaccharides on lutropin, follitropin, and thyrotropin. II. Distributions of sulfated and sialylated oligosaccharides on bovine, ovine, and human pituitary glycoprotein hormones. J Biol Chem. (1988) 263:36-44.

3. Walton WJ, Nguyen VT, Butnev VY, Singh V, Moore WT, Bousfield GR. Characterization of human FSH isoforms reveals a nonglycosylated betasubunit in addition to the conventional glycosylated beta-subunit. $J$ Clin Endocrinol Metab. (2001) 86:3675-85. doi: 10.1210/jcem.86.8.7712

4. Bousfield GR, May JV, Davis JS, Dias JA, Kumar TR. In vivo and in vitro impact of carbohydrate variation on human follicle-stimulating hormone function. Front Endocrinol (Lausanne) (2018) 9:216. doi: 10.3389/fendo.2018. 00216

5. Huhtaniemi IT, Themmen AP, Mutations in human gonadotropin and gonadotropin-receptor genes. Endocrine (2005) 26:207-17. doi: 10.1385/ENDO:26:3:207

6. Jonas KC, Oduwole OO, Peltoketo H, Rulli SB, Huhtaniemi IT. Mouse models of altered gonadotrophin action: insight into male reproductive disorders, Reproduction (2014) 148:R63-70. doi: 10.1530/REP-1 4-0302

7. Stilley JA, Guan R, Santillan DA, Mitchell BF, Lamping KG, Segaloff DL. Differential regulation of human and mouse myometrial contractile activity by FSH as a function of FSH receptor density. Biol Reprod. (2016) 95:36. doi: 10.1095/biolreprod.116.141648

8. Liu P, Ji Y, Yuen T, Rendina-Ruedy E, DeMambro VE, Dhawan S, et al. Blocking FSH induces thermogenic adipose tissue and reduces body fat. Nature (2017) 546:107-12. doi: 10.1038/nature22342
In summary, our understanding of the complexity of GPCR signaling pathways via the tight control of their intracellular location has been advanced through studies on the gonadotropin hormone receptors. Such mechanisms have highlighted the interconnected nature of these intracellular systems, and thus a primary future goal is to further understand the significance of these molecular systems to health and disease if they are to be of therapeutic value. The critical nature of intracellular sorting of FSHR to signaling has been demonstrated, so it is not a question of is it important, but rather how the intricacies of modulating receptor from one intracellular compartment to another impact specific functions in vivo. This would provide the opportunity to be able to target these intracellular signaling modalities with high precision, in order to create the next generation of therapeutics for reproductive medicine.

\section{AUTHOR CONTRIBUTIONS}

All authors listed have made a substantial, direct and intellectual contribution to the work, and approved it for publication.

\section{ACKNOWLEDGMENTS}

This work was supported by grants from the Genesis Research Trust (P15844, P67019) and Wellcome Trust (WT087248MA) to $\mathrm{AH}$ and an Imperial College London President's scholarship to NS.

9. Sun L, Peng Y, Sharrow AC, Iqbal J, Zhang Z, Papachristou DJ, et al. FSH directly regulates bone mass. Cell (2006) 125:247-60. doi: 10.1016/j.cell.2006.01.051

10. Liu XM, Chan HC, Ding GL, Cai J, Song Y, Wang TT, et al. FSH regulates fat accumulation and redistribution in aging through the Galphai/Ca(2+)/CREB pathway. Aging Cell (2015) 14:409-20. doi: 10.1111/acel.12331

11. Puett D, Li Y, DeMars G, Angelova K, Fanelli F. A functional transmembrane complex: the luteinizing hormone receptor with bound ligand and $\mathrm{G}$ protein. Mol Cell Endocrinol. (2007) 260-262:126-36. doi: 10.1016/j.mce.2006.05.009

12. Thomas RM, Nechamen CA, Mazurkiewicz JE, Ulloa-Aguirre A, Dias JA. The adapter protein APPL1 links FSH receptor to inositol 1,4,5trisphosphate production and is implicated in intracellular $\mathrm{Ca}(2+)$ mobilization. Endocrinology (2011) 152:1691-701. doi: 10.1210/en.2010-1353

13. Hirsch B, Kudo M, Naro F, Conti M, Hsueh AJ. The C-terminal third of the human luteinizing hormone $(\mathrm{LH})$ receptor is important for inositol phosphate release: analysis using chimeric human $\mathrm{LH} /$ follicle-stimulating hormone receptors. Mol Endocrinol. (1996) 10:1127-37.

14. Zhu X, Gilbert S, Birnbaumer M, Birnbaumer L. Dual signaling potential is common among Gs-coupled receptors and dependent on receptor density. Mol Pharmacol. (1994) 46:460-9.

15. Lin YF, Tseng MJ, Hsu HL, Wu YW Lee YH, Tsai YH. A novel follicle-

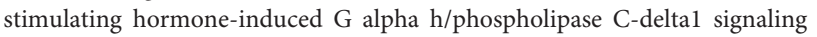
pathway mediating rat sertoli cell Ca2+-influx. Mol Endocrinol. (2006) 20:2514-27. doi: 10.1210/me.2005-0347

16. Crepieux P, Marion S, Martinat N, Fafeur V, Vern YL, Kerboeuf D, et al. The ERK-dependent signalling is stage-specifically modulated by FSH, during primary Sertoli cell maturation. Oncogene (2001) 20:4696-709. doi: $10.1038 /$ sj.onc. 1204632

17. Jonas KC, Hanyaloglu AC. Impact of $G$ protein-coupled receptor heteromers in endocrine systems. Mol Cell Endocrinol. (2017) 449:21-7. doi: 10.1016/j.mce.2017.01.030 
18. Ferre S, Casado V, Devi LA, Filizola M, Jockers R, Lohse MJ, et al. G proteincoupled receptor oligomerization revisited: functional and pharmacological perspectives, Pharmacol Rev. (2014) 66:413-34. doi: 10.1124/pr.113.008052

19. Patel HH, Murray F, Insel PA. G-protein-coupled receptor-signaling components in membrane raft and caveolae microdomains. Handb Exp Pharmacol. (2008) (186):167-84. doi: 10.1007/978-3-540-72843-6_7

20. Feng X, Zhang M, Guan R, Segaloff DL. Heterodimerization between the lutropin and follitropin receptors is associated with an attenuation of hormone-dependent signaling, Endocrinology (2013) 154:3925-30. doi: 10.1210/en.2013-1407

21. Jiang X, Fischer D, Chen X, McKenna SD, Liu H, Sriraman V, et al. Evidence for follicle-stimulating hormone receptor as a functional trimer. J Biol Chem. (2014) 289:14273-82. doi: 10.1074/jbc.M114.549592

22. Li X, Chen W, Li P, Wei J, Cheng Y, Liu P, et al. Follicular stimulating hormone accelerates atherogenesis by increasing endothelial VCAM-1 expression. Theranostics (2017) 7:4671-4688. doi: 10.7150/thno.21216

23. Wilden U, Wust E, Weyand I, Kuhn H. Rapid affinity purification of retinal arrestin (48 $\mathrm{kDa}$ protein) via its light-dependent binding to phosphorylated rhodopsin. FEBS Lett. (1986) 207:292-5. doi: 10.1016/0014-5793(86)81507-1

24. Craft CM, Whitmore DH, Wiechmann AF. Cone arrestin identified by targeting expression of a functional family. J Biol Chem. (1994) 269:4613-9.

25. Attramadal H, Arriza JL, Aoki C, Dawson TM, Codina J, Kwatra MM, et al. Beta-arrestin2, a novel member of the arrestin/beta-arrestin gene family. J Biol Chem. (1992) 267:17882-90.

26. Lohse MJ, Benovic JL, Codina J, Caron MG, Lefkowitz RJ. beta-Arrestin: a protein that regulates beta-adrenergic receptor function. Science (1990) 248:1547-50. doi: 10.1126/science. 2163110

27. Rajagopal S, Shenoy SK. GPCR desensitization: acute and prolonged phases. Cell Signal. (2018) 41:9-16. doi: 10.1016/j.cellsig.2017.01.024

28. Reiter E, Ayoub MA, Pellissier LP, Landomiel F, Musnier A, Trefier A, et al. beta-arrestin signalling and bias in hormone-responsive GPCRs. Mol Cell Endocrinol. (2017) 449:28-41. doi: 10.1016/j.mce.2017.01.052

29. Liggett SB. Phosphorylation barcoding as a mechanism of directing GPCR signaling. Sci Signal. (2011) 4:pe36. doi: 10.1126/scisignal.2002331

30. Shukla AK, Westfield GH, Xiao K, Reis RI, Huang LY, Tripathi-Shukla P, et al. Visualization of arrestin recruitment by a G-protein-coupled receptor. Nature (2014) 512:218-22. doi: 10.1038/nature13430

31. Thomsen ARB, Plouffe B, Cahill TJ, Shukla AK, Tarrasch JT, Dosey AM, et al. GPCR-G protein-beta-arrestin super-complex mediates sustained G protein signaling. Cell (2016) 166:907-919. doi: 10.1016/j.cell.2016.07.004

32. Cahill TJ III, Thomsen AR, Tarrasch JT, Plouffe B, Nguyen AH, Yang F, et al. Distinct conformations of GPCR-beta-arrestin complexes mediate desensitization, signaling, and endocytosis. Proc Natl Acad Sci USA. (2017) 114:2562-2567. doi: 10.1073/pnas.1701529114

33. Eichel K, Jullie D, Barsi-Rhyne B, Latorraca NR, Masureel M, Sibarita JB, et al. Catalytic activation of beta-arrestin by GPCRs. Nature (2018) 557:381-386. doi: 10.1038/s41586-018-0079-1

34. Ranjan R, Dwivedi H, Baidya M, Kumar M, Shukla AK. Novel Structural insights into GPCR-beta-arrestin interaction and signaling. Trends Cell Biol. (2017) 27:851-862. doi: 10.1016/j.tcb.2017.05.008

35. Troispoux C, Guillou F, Elalouf JM, Firsov D, Iacovelli L, De Blasi A, et al. Involvement of $\mathrm{G}$ protein-coupled receptor kinases and arrestins in desensitization to follicle-stimulating hormone action. Mol Endocrinol. (1999) 13:1599-614. doi: 10.1210/mend.13.9.0342

36. Kara E, Crepieux P, Gauthier C, Martinat N, Piketty V, Guillou F, et al. A phosphorylation cluster of five serine and threonine residues in the C-terminus of the follicle-stimulating hormone receptor is important for desensitization but not for beta-arrestin-mediated ERK activation. Mol Endocrinol. (2006) 20:3014-26. doi: 10.1210/me.2006-0098

37. Bhaskaran RS, Min L, Krishnamurthy H, Ascoli M. Studies with chimeras of the gonadotropin receptors reveal the importance of third intracellular loop threonines on the formation of the receptor/nonvisual arrestin complex. Biochemistry (2003) 42:13950-13959. doi: 10.1021/bi034907w

38. Kishi H, Ascoli M. Multiple distant amino acid residues present in the serpentine region of the follitropin receptor modulate the rate of agonist-induced internalization. J Biol Chem. (2000) 275:31030-7. doi: 10.1074/jbc.M005528200
39. Kumari P, Srivastava A, Banerjee R, Ghosh E, Gupta P, Ranjan R, et al. Functional competence of a partially engaged GPCR-beta-arrestin complex. Nat Commun. (2016) 7:13416. doi: 10.1038/ncomms13416

40. McDonald PH, Chow CW, Miller WE, Laporte SA, Field ME, Lin FT, et al. Beta-arrestin 2: a receptor-regulated MAPK scaffold for the activation of JNK3. Science (2000) 290:1574-7. doi: 10.1126/science.290.5496.1574

41. DeFea KA, Zalevsky J, Thoma MS, Dery O, Mullins RD, Bunnett NW. betaarrestin-dependent endocytosis of proteinase-activated receptor 2 is required for intracellular targeting of activated ERK1/2. J Cell Biol. (2000) 148:1267-81. doi: $10.1083 /$ jcb.148.6.1267

42. Terrillon S, Bouvier M. Receptor activity-independent recruitment of betaarrestin2 reveals specific signalling modes. EMBO J. (2004) 23:3950-61. doi: 10.1038/sj.emboj.7600387

43. Ayoub MA, Landomiel F, Gallay N, Jegot G, Poupon A, Crepieux P, et al. Assessing gonadotropin receptor function by resonance energy transfer-based assays. Front Endocrinol (Lausanne) (2015) 6:130. doi: $10.3389 /$ fendo.2015.00130

44. Jean-Alphonse FG, Bowersox S, Chen S, Beard G, Puthenveedu MA, Hanyaloglu AC. Spatially restricted G protein-coupled receptor activity via divergent endocytic compartments. J Biol Chem. (2014) 289:3960-77. doi: 10.1074/jbc.M113.526350

45. Piketty V, Kara E, Guillou F, Reiter E, Crepieux P. Follicle-stimulating hormone (FSH) activates extracellular signal-regulated kinase phosphorylation independently of beta-arrestin- and dynamin-mediated FSH receptor internalization. Reprod Biol Endocrinol. (2006) 4:33. doi: 10.1186/1477-7827-4-33

46. Eichel K, Jullie D, von Zastrow M. beta-Arrestin drives MAP kinase signalling from clathrin-coated structures after GPCR dissociation. Nat Cell Biol. (2016) 18:303-10. doi: 10.1038/ncb3307

47. Nakamura K, Lazari MF, Li S, Korgaonkar C, Ascoli M. Role of the rate of internalization of the agonist-receptor complex on the agonist-induced downregulation of the lutropin/choriogonadotropin receptor. Mol Endocrinol. (1999) 13:1295-304. doi: 10.1210/mend.13.8.0331

48. Kishi H, Krishnamurthy H, Galet C, Bhaskaran RS, Ascoli M. Identification of a short linear sequence present in the C-terminal tail of the rat follitropin receptor that modulates arrestin-3 binding in a phosphorylation-independent fashion. J Biol Chem. (2002) 277:21939-46. doi: 10.1074/jbc.M110894200

49. Trefier A, Musnier A, Landomiel F, Bourquard T, Boulo T, Ayoub MA, et al. G protein-dependent signaling triggers a beta-arrestin-scaffolded p70S6K/ rpS6 module that controls 5'TOP mRNA translation. FASEB J. (2018) 32:11541169. doi: 10.1096/fj.201700763R

50. Latorraca NR, Wang JK, Bauer B, Townshend RJL, Hollingsworth SA, Olivieri JE, et al. Molecular mechanism of GPCR-mediated arrestin activation. Nature (2018) 557:452-456. doi: 10.1038/s41586-018-0077-3

51. Landomiel F, Gallay N, Jégot G, Tranchant T, Durand G, Bourquard T, et al. Biased signalling in follicle stimulating hormone action. Mol Cell Endocrinol. (2014) 1:452-459. doi: 10.1016/j.mce.2013.09.035

52. Grundmann M, Merten N, Malfacini D, Inoue A, Preis P, Simon K, et al. Lack of beta-arrestin signaling in the absence of active G proteins, Nat Commun. (2018) 1:341. doi: 10.1038/s41467-017-02661-3

53. O’Hayre M, Eichel K, Avino S, Zhao X, Steffen DJ, Feng X, et al. Genetic evidence that beta-arrestins are dispensable for the initiation of beta2-adrenergic receptor signaling to ERK. Sci Signal. (2017) 10:eaal3395. doi: 10.1126/scisignal.aal3395

54. Tranchant T, Durand G, Gauthier C, Crepieux P, Ulloa-Aguirre A, Royere D, et al. Preferential beta-arrestin signalling at low receptor density revealed by functional characterization of the human FSH receptor A189 V mutation. Mol Cell Endocrinol. (2011) 1:109-18. doi: 10.1016/j.mce.2010.08.016

55. Aittomaki K, Lucena JL, Pakarinen P, Sistonen P, Tapanainen J, Gromoll $\mathrm{J}$, et al. Mutation in the follicle-stimulating hormone receptor gene causes hereditary hypergonadotropic ovarian failure. Cell (1995) 1:959-68. doi: 10.1016/0092-8674(95)90275-9

56. Miyoshi T, Otsuka F, Shimasaki S. GRK-6 mediates FSH action synergistically enhanced by estrogen and the oocyte in rat granulosa cells. Biochem Biophys Res Commun. (2013) 1:401-6. doi: 10.1016/j.bbrc.2013.04.002

57. Casarini L, Reiter E, Simoni M. beta-arrestins regulate gonadotropin receptormediated cell proliferation and apoptosis by controlling different FSHR or 
LHCGR intracellular signaling in the hGL5 cell line. Mol Cell Endocrinol. (2016) 437:11-21. doi: 10.1016/j.mce.2016.08.005

58. Hanyaloglu AC, von Zastrow M. Regulation of GPCRs by endocytic membrane trafficking and its potential implications. Annu Rev Pharmacol Toxicol. (2008) 48:537-68. doi: 10.1146/annurev.pharmtox.48.113006.094830

59. Irannejad R, Tsvetanova NG, Lobingier BT, von Zastrow M. Effects of endocytosis on receptor-mediated signaling. Curr Opin Cell Biol. (2015) 35:137-43. doi: 10.1016/j.ceb.2015.05.005

60. Sposini S, Hanyaloglu AC. Spatial encryption of G protein-coupled receptor signaling in endosomes; mechanisms and applications. Biochem Pharmacol. (2017) 143:1-9. doi: 10.1016/j.bcp.2017.04.028

61. Pavlos NJ, Friedman PA. GPCR Signaling and trafficking: the long and short of It. Trends Endocrinol Metab. (2017) 1:213-26. doi: 10.1016/j.tem.2016.10.007

62. Bowman SL, Puthenveedu MA. Postendocytic Sorting of adrenergic and opioid receptors: new mechanisms and functions. Prog Mol Biol Transl Sci. (2015) 132:189-206. doi: 10.1016/bs.pmbts.2015.03.005

63. Sposini S, Hanyaloglu AC. Evolving view of membrane trafficking and signaling systems for g protein-coupled receptors. Prog Mol Subcell Biol. (2018) 57:273-99. doi: 10.1007/978-3-319-96704-2_10

64. Hanyaloglu AC. Advances in membrane trafficking and endosomal signaling of g protein-coupled receptors. Int Rev Cell Mol Biol. (2018) 339:93-131. doi: 10.1016/bs.ircmb.2018.03.001

65. Hislop JN, von Zastrow M. Role of ubiquitination in endocytic trafficking of G-protein-coupled receptors. Traffic (2011) 1:137-48. doi: $10.1111 / j .1600-0854.2010 .01121 . x$

66. Kennedy JE, Marchese A, Regulation of GPCR Trafficking by Ubiquitin. Prog Mol Biol Transl Sci. (2015) 132:15-38. doi: 10.1016/bs.pmbts.2015.02.005

67. Cao TT, Deacon HW, Reczek D, Bretscher A, von Zastrow M. A kinase-regulated PDZ-domain interaction controls endocytic sorting of the beta2-adrenergic receptor. Nature (1999) 401:286-90. doi: 10.1038/ 45816

68. Hirakawa T, Galet C, Kishi M, Ascoli M. GIPC binds to the human lutropin receptor (hLHR) through an unusual PDZ domain binding motif, and it regulates the sorting of the internalized human choriogonadotropin and the density of cell surface hLHR. J Biol Chem. (2003) 1:49348-57. doi: 10.1074/jbc.M306557200

69. He J, Bellini M, Inuzuka H, Xu J, Xiong Y, Yang X, et al. Proteomic analysis of betal-adrenergic receptor interactions with PDZ scaffold proteins. J Biol Chem. (2006) 1:2820-7. doi: 10.1074/jbc.M509503200

70. Lauffer BE, Melero C, Temkin P, Lei C, Hong W, Kortemme T, et al. SNX27 mediates PDZ-directed sorting from endosomes to the plasma membrane. $J$ Cell Biol. (2010) 1:565-74. doi: 10.1083/jcb.201004060

71. Marchese A, Paing MM, Temple BR, Trejo J. G protein-coupled receptor sorting to endosomes and lysosomes. Annu Rev Pharmacol Toxicol. (2008) 48:601-29. doi: 10.1146/annurev.pharmtox.48.113006.094646

72. Krishnamurthy H, Kishi H, Shi M, Galet C, Bhaskaran RS, Hirakawa $\mathrm{T}$, et al. Postendocytotic trafficking of the follicle-stimulating hormone (FSH)-FSH receptor complex. Mol Endocrinol. (2003) 1:2162-76. doi: 10.1210/me.2003-0118

73. Melo-Nava B, Casas-Gonzalez P, Perez-Solis MA, Castillo-Badillo J, Maravillas-Montero JL, Jardon-Valadez E, et al. Role of cysteine residues in the carboxyl-terminus of the follicle-stimulating hormone receptor in intracellular traffic and postendocytic processing. Front Cell Dev Biol. (2016) 4:76. doi: $10.3389 /$ fcell.2016.00076

74. Uribe A, Zarinan T, Perez-Solis MA, Gutierrez-Sagal R, Jardon-Valadez E, Pineiro A, et al. Functional and structural roles of conserved cysteine residues in the carboxyl-terminal domain of the follicle-stimulating hormone receptor in human embryonic kidney 293 cells. Biol Reprod. (2008) 1:869-82. doi: 10.1095/biolreprod.107.063925

75. Hu LA, Chen W, Martin NP, Whalen EJ, Premont RT, Lefkowitz RJ. GIPC interacts with the betal-adrenergic receptor and regulates beta1adrenergic receptor-mediated ERK activation. J Biol Chem. (2003) 1:26295301. doi: 10.1074/jbc.M212352200

76. Nechamen CA, Thomas RM, Cohen BD, Acevedo G, Poulikakos PI, Testa $\mathrm{JR}$, et al. Human follicle-stimulating hormone (FSH) receptor interacts with the adaptor protein APPL1 in HEK 293 cells: potential involvement of the PI3K pathway in FSH signaling. Biol Reprod. (2004) 1:629-36. doi: 10.1095/biolreprod.103.025833
77. Nechamen CA, Thomas RM, Dias JA, APPL1, APPL2, Akt2 and FOXO1a interact with FSHR in a potential signaling complex. Mol Cell Endocrinol. (2007) 1:93-9. doi: 10.1016/j.mce.2006.08.014

78. Lin DC, Quevedo C, Brewer NE, Bell A, Testa JR, Grimes ML, et al. APPL1 associates with TrkA and GIPCl and is required for nerve growth factor-mediated signal transduction. Mol Cell Biol. (2006) 1:8928-41. doi: 10.1128/MCB.00228-06

79. Liu Z, Xiao T, Peng X, Li G, Hu F. APPLs: more than just adiponectin receptor binding proteins. Cell Signal (2017) 32:76-84. doi: 10.1016/j.cellsig.2017.01.018

80. Zoncu R, Perera RM, Balkin DM, Pirruccello M, Toomre D, De Camilli P, A phosphoinositide switch controls the maturation and signaling properties of APPL endosomes. Cell (2009) 1:1110-21. doi: 10.1016/j.cell.2009.01.032

81. Kalaidzidis I, Miaczynska M, Brewinska-Olchowik M, Hupalowska A, Ferguson C, Parton RG, et al. APPL endosomes are not obligatory endocytic intermediates but act as stable cargo-sorting compartments. J Cell Biol. (2015) 1:123-44. doi: 10.1083/jcb.201311117

82. Diggins NL, Webb DJ, APPL1 is a multifunctional endosomal signaling adaptor protein. Biochem Soc Trans. (2017) 1:771-9. doi: 10.1042/BST20160191

83. Dias JA, Mahale SD, Nechamen CA, Davydenko O, Thomas RM, Ulloa-Aguirre A, Emerging roles for the FSH receptor adapter protein APPL1 and overlap of a putative 14-3-3tau interaction domain with a canonical G-protein interaction site. Mol Cell Endocrinol. 329 (2010) 17-25. doi: 10.1016/j.mce.2010.05.009

84. Sposini S, Jean-Alphonse FG, Ayoub MA, Oqua A, West C, Lavery $S$, et al. Integration of GPCR signaling and sorting from very early endosomes via opposing APPL1 Mechanisms. Cell Rep. (2017) 1:2855-2867. doi: 10.1016/j.celrep.2017.11.023

85. Irannejad R, Tomshine JC, Tomshine JR, Chevalier M, Mahoney JP, Steyaert $\mathrm{J}$, et al. Conformational biosensors reveal GPCR signalling from endosomes. Nature (2013) 495:534-8. doi: 10.1038/nature12000

86. Feinstein TN, Wehbi VL, Ardura JA, Wheeler DS, Ferrandon S, Gardella TJ, et al. Retromer terminates the generation of cAMP by internalized PTH receptors. Nat Chem Biol. (2011) 1:278-84. doi: 10.1038/nchembio.545

87. Feinstein TN, Yui N, Webber MJ, Wehbi VL, Stevenson HP, King JD, Jr., et al. Noncanonical control of vasopressin receptor type 2 signaling by retromer and arrestin. J Biol Chem. (2013) 1:27849-60. doi: 10.1074/jbc.M112.445098

88. Lobingier BT, Huttenhain R, Eichel K, Miller KB, Ting AY, von Zastrow M, et al. An approach to spatiotemporally resolve protein interaction networks in living cells. Cell (2017) 1:350-360 e12. doi: 10.1016/j.cell.2017.03.022

89. Comim FV, Hardy K, Franks S. Adiponectin and its receptors in the ovary: further evidence for a link between obesity and hyperandrogenism in polycystic ovary syndrome. PLoS ONE (2013) 1:e80416. doi: 10.1371/journal.pone.0080416

90. Lyga S, Volpe S, Werthmann RC, Gotz K, Sungkaworn T, Lohse MJ, et al. Persistent cAMP Signaling by internalized $\mathrm{lh}$ receptors in ovarian follicles. Endocrinology (2016) 1:1613-21. doi: 10.1210/en.2015-1945

91. Casas-Gonzalez P, Scaglia HE, Perez-Solis MA, Durand G, Scaglia J, Zarinan T, et al. Normal testicular function without detectable follicle-stimulating hormone. A novel mutation in the follicle-stimulating hormone receptor gene leading to apparent constitutive activity and impaired agonist-induced desensitization and internalization. Mol Cell Endocrinol. (2012) 364:71-82. doi: 10.1016/j.mce.2012.08.011

92. Nordhoff V, Sonntag B, von Tils D, Gotte M, Schuring AN, Gromoll J, et al. Effects of the FSH receptor gene polymorphism p.N680S on cAMP and steroid production in cultured primary human granulosa cells. Reprod Biomed Online (2011) 1:196-203. doi: 10.1016/j.rbmo.2011.04.009

93. Casarini L, Moriondo V, Marino M, Adversi F, Capodanno F, Grisolia C, et al. FSHR polymorphism p.N680S mediates different responses to FSH in vitro. Mol Cell Endocrinol. (2014) 393:83-91. doi: 10.1016/j.mce.2014.06.013

94. Dominguez-Lopez P, Diaz-Cueto L, Arechavaleta-Velasco M, CaldinoSoto F, Ulloa-Aguirre A, Arechavaleta-Velasco F. The follicle-stimulating hormone receptor Asn680Ser polymorphism is associated with preterm birth in Hispanic women. J Matern Fetal Neonatal Med. (2018) 1:580-5. doi: 10.1080/14767058.2017.1292245

95. Simoni M, Santi D, Negri L, Hoffmann I, Muratori M, Baldi E, et al. Treatment with human, recombinant FSH improves sperm DNA 
fragmentation in idiopathic infertile men depending on the FSH receptor polymorphism p.N680S: a pharmacogenetic study. Hum Reprod. (2016) 1:1960-9. doi: 10.1093/humrep/dew167

96. Jensen DD, Lieu T, Halls ML, Veldhuis NA, Imlach WL, Mai QN, et al. Neurokinin 1 receptor signaling in endosomes mediates sustained nociception and is a viable therapeutic target for prolonged pain relief. Sci Transl Med. (2017) 9:eaal3447. doi: 10.1126/scitranslmed.aal3447

97. Yarwood RE, Imlach WL, Lieu T, Veldhuis NA, Jensen DD, Klein Herenbrink $\mathrm{C}$, et al. Endosomal signaling of the receptor for calcitonin gene-related peptide mediates pain transmission. Proc Natl Acad Sci USA. (2017) 1:12309-14. doi: 10.1073/pnas.1706656114

98. Jimenez-Vargas NN, Pattison LA, Zhao P, Lieu T, Latorre R, Jensen DD, et al. Protease-activated receptor-2 in endosomes signals persistent pain of irritable bowel syndrome. Proc Natl Acad Sci USA. (2018) 1:E7438-7447. doi: $10.1073 /$ pnas. 1721891115
99. Nataraja SG, Yu HN, Palmer SS. Discovery and development of small molecule allosteric modulators of glycoprotein hormone receptors. Front Endocrinol. (2015) 6:142. doi: 10.3389/fendo.2015. 00142

Conflict of Interest Statement: The authors declare that the research was conducted in the absence of any commercial or financial relationships that could be construed as a potential conflict of interest.

Copyright $\odot 2018$ Sayers and Hanyaloglu. This is an open-access article distributed under the terms of the Creative Commons Attribution License (CC BY). The use, distribution or reproduction in other forums is permitted, provided the original author(s) and the copyright owner(s) are credited and that the original publication in this journal is cited, in accordance with accepted academic practice. No use, distribution or reproduction is permitted which does not comply with these terms. 